\title{
A Dynamic 3-D Cardiac Surface Model from MR Images
}

\author{
B Delhay ${ }^{1}$, J Lötjönen ${ }^{2}$, P Clarysse $^{1}$, T Katila $^{3}$, IE Magnin ${ }^{1}$ \\ ${ }^{1}$ Creatis, CNRS UMR \#5515, INSERM U630, F-69621 Villeurbanne Cedex, France \\ ${ }^{2}$ VTT Information Technology, POBox 1206, 33101 Tampere, Finland \\ ${ }^{3}$ Laboratory of Biomedical Engineering, Helsinki University of Technology, PO Box 2200, 02015 \\ HUT, Finland
}

\begin{abstract}
Cardiac 3D + time segmentation and motion estimation are recognized as difficult prerequisite tasks for any quantitative analysis of cardiac images. Some recent algorithms aim to consider a temporal constraint to increase the accuracy of results. To improve the temporal consistency, prior knowledge about cardiac dynamics can be used. In this paper, we propose to build a new Statistical Dynamic Model (SDM) of the heart by learning through a population of healthy individuals. This SDM is composed by a set of semi-landmarks which describe the heart surfaces. For each of them, a mean trajectory and variability around it are derived. The SDM provides a reasonable constraint for a temporally regularized segmentation and motion tracking algorithm.
\end{abstract}

\section{Introduction}

Due to the great progress of image acquisition devices, it is now possible to better explore the dynamics of moving organs such as the heart and the lungs. Magnetic Resonance Imaging (MRI) and Multiple Detector Computed Tomography (MDCT) can provide meaningful information about the 3D anatomy and contractile function of the heart. 3D+time segmentations and motion estimations from tomographic cardiac images are recognized as difficult prerequisite tasks for any quantitative analysis of cardiac functions. In [1], the need for a temporal constraint is claimed to increase the accuracy of segmentations results. However, actual dynamic cardiac segmentations and motion estimators still suffer from a lack of temporal consistency because they don't take into account the true behavior of cardiac structures.

Some recently introduced models try to incorporate dynamics features of patient-specific ventricles and myocardium [2, 3]. However, this kind of approach does not consider the large variability of patients' heart dynamics within a population of individuals.

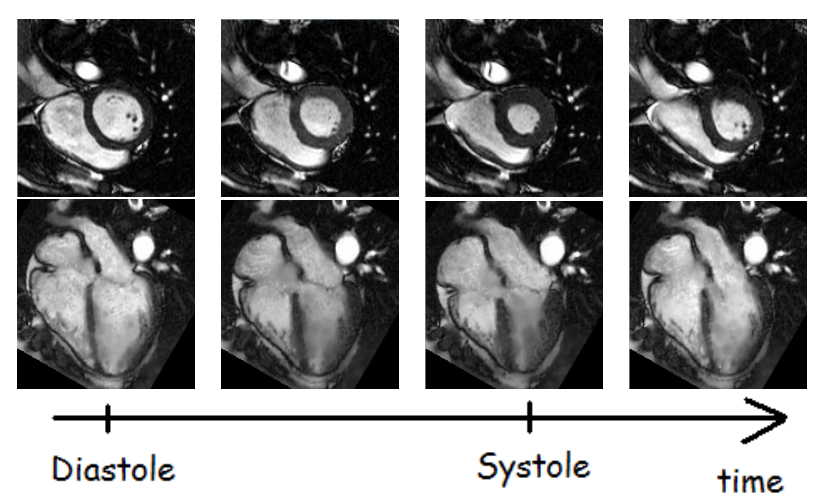

Figure 1. Cine MRI sequences. Top, short axis slices. Bottom, 4 cavities long axis slices.

In this paper, a statistical a priori model of the spatiotemporal evolution of cardiac structures (ventricles and atria) is built. Such a model can be very valuable to constrain automatic segmentations and motion estimations. Our model was built from short- and long-axis cine MRI acquisitions of 16 healthy subjects to represent the statistical distribution of identified shape semi-landmarks in space and over time. We also give a way to introduce our model in a segmentation and motion tracking algorithm.

\section{Material and method}

\subsection{Data}

Cine-MR Image sequences of 16 healthy voluntary subjects formed the reference dataset. In the sequel, we will denote $N=16$, the number of subjects. For each of them, both Short Axis (SA) and Long Axis (LA) images have been acquired.

The images were obtained with a $1.5 \mathrm{~T}$ Siemens Vision System at the Helsinki Medical Imaging Center (Helsinki University Central Hospital, HUCH). Cine Images were acquired using breathhold and ECG-triggered protocol. For the volumetric study of ventricles, SA slice thickness 
was $6-7 \mathrm{~mm}$ with 7-8 mm gap. Cine images in the four chamber planes with 6-7 mm slice thickness and 3-4 mm gap were acquired for atria reconstruction. The number of SA and LA slices was 4-5 and 4-7, respectively, depending on the size of the subjects heart. The pixel size for SA and LA images was either $1.0 \times 1.0 \mathrm{~mm}$ or $1.4 \times 1.4 \mathrm{~mm}$.

The following time resolution parameters were used: repetition time $30-40 \mathrm{~ms}$, echo time $4.8 \mathrm{~ms}$, flip angle $20^{\circ}$, matrix size $256 \times 256$ and field of view $250-300 \mathrm{~mm}$. All the sequences cover the cardiac cycle within 22-29 acquisitions depending on the subject's beating rate.

All the images in the data set were preprocessed using in slice motion compensation [4] and shaped-based interpolation [5] to provide SA and LA volume sequences with reduced motion artifacts. For more details see [6].

\subsection{Static model construction}

In [6], a static 3-D cardiac surface model was built. To this aim, the first image of each time series, i.e. the enddiastolic (ED) phase, was considered as the reference image. This time point was chosen because heart motion is minimal during this stage and motion artifacts in ED images might be reduced. Let $V^{j}, j=\{0 \ldots N-1\}$ be the set of reference ED images.

For each case, the four chambers and the pericardium were manually segmented from both SA and LA reference images by a medical expert. Affine registrations between all the reference images were used to build a mean surface shape model $\overline{\mathcal{S}}$ (Fig.(2)). Each node $P_{i}$ of the resulting triangulated surface is considered as a semi-landmark (2086 at total).

$$
\overline{\mathcal{S}}=\left\{P_{i}\right\} \quad \text { with } \quad i=[0 . .2085]
$$

An intensity image, $\bar{V}$, was constructed from the mean shape. To propagate $\overline{\mathcal{S}}$ to the database subjects, volumetric transformations $T^{j}$, between $\bar{V}$ and $V^{j}$ were estimated using a non-rigid registration algorithm [7]. Every transformations $V^{j}$ were applied to $\overline{\mathcal{S}}$. As a result, a set of corresponding semi-landmarks, $\mathcal{S}_{j}$, was obtained for all database subjects.

$$
\mathcal{S}_{j}=V^{j}(\overline{\mathcal{S}})
$$

\subsection{Dynamic model construction}

\subsubsection{D dataset segmentation}

As in $[2,3]$, for each data set $j$, a non-rigid registrations, was applied to extract volumetric transformations $T_{t_{k}}^{j}$, between the ED time phase volume $\left(V^{j}\right)$ and all the remaining time phase volumes $\left(V_{t_{k}}^{j}\right)$ in a $4 \mathrm{D}$ data set (Fig.(3)).
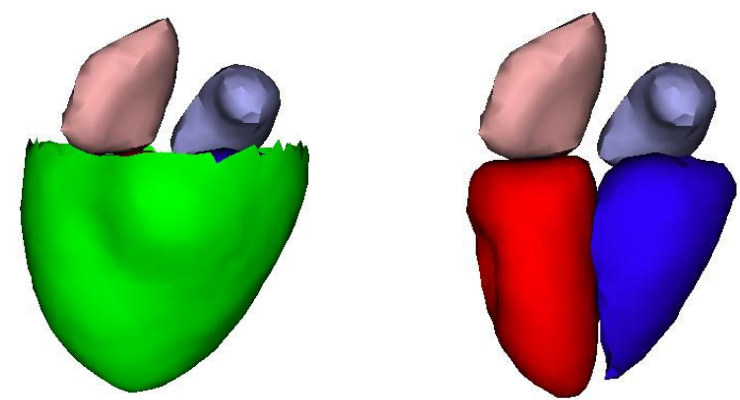

Figure 2. Geometrical Mean Model with (left), and without pericardium (right)

Thus, sequential cardiac structure meshes were found by warping $\mathcal{S}_{j}$ according to those estimated volumetric transformations. Finally, the 3-D transformations were manually corrected to accurately refine the segmentations. As a result, sequential segmentations for 23-29 time points, depending on the subject, were produced for each of the 16 subjects.

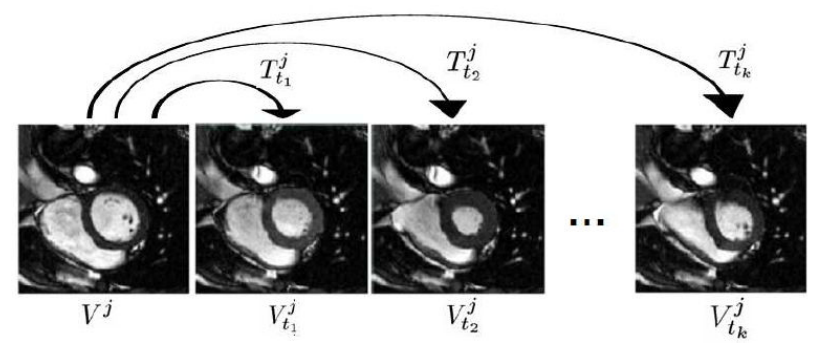

Figure 3. Volumetric transformation estimations between the ED time phase volume $\left(V^{j}\right)$ and all the remaining time phase volumes $\left(V_{t_{k}}^{j}\right)$ for the subject $j$

\subsubsection{Time resampling}

For each data set $j$, a semi-landmark $P_{i}$ of $\mathcal{S}_{j}$ describes a $3 \mathrm{D}$ trajectory. However, the number of phases and the systole time points differs from one subjects to the other. Therefore, in order to construct the dynamic cardiac surface model, the trajectories are resampled to obtain unified temporally consistent semi-landmarks paths. To this aim, spline interpolation provides smooth trajectories (Fig.(4): dotted line), which are then resampled according to the detected systolic time frame (Fig.(4): dashed line). The cardiac cycle is ranged between 0 and 1 and the end systolic time point was set to $1 / 3$ the cardiac cycle. 


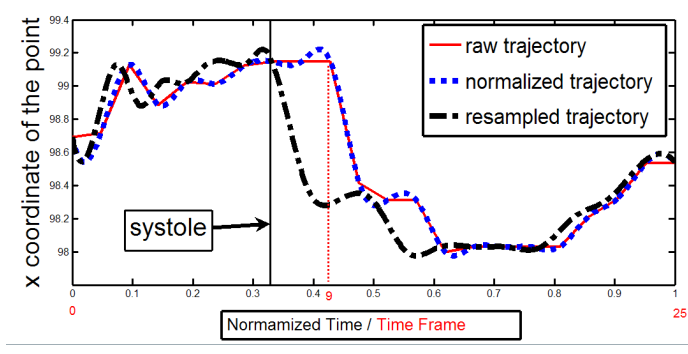

Figure 4. X coordinate of one semi-landmark's trajectories during the resampling stage. The Straight line displays the raw trajectory which is the result of all successive segmentations. The Dotted line shows interpolated smooth trajectory and the Dashed line represents resampled trajectory

\subsubsection{Statistical dynamic model}

A mean model $\overline{\mathcal{D}}$ which describes a mean trajectory for each semi-landmark $P_{i}$, is built from resampled trajectories and affine transformations.

$$
\overline{\mathcal{D}}=\left\{P_{i}(t)\right\} \quad \text { with } i=[0 . .2085] \text { and } t=[0 . .1]
$$

The variability in the semi-landmark location is modeled using statistics where each $P_{i}$ is considered as a random vector:

$$
\left\{P_{i}(t)\right\}=\left\{\mathbf{X}_{i}(t)\right\}=\left\{X_{i}(t), Y_{i}(t), Z_{i}(t)\right\}
$$

We consider that $x_{i_{j}}(t), y_{i_{j}}(t)$ and $z_{i_{j}}(t)$ are the instances of the random variables $X_{i}(t), Y_{i}(t)$ and $Z_{i}(t)$ binded to the $j^{t h}$ case in the data set. Non-linear probability distributions are approximated from $N$ samples using Parzen windowing to realistically model the variability of each semi-landmark. As a result, dynamic probability density functions $p\left(\mathbf{X}_{i}\left(t_{k}\right)=\mathbf{x}_{i}\left(t_{k}\right)\right)$ are the likelihoods that the spatio-temporal semi-landmark $P_{i}$ is at the 3D coordinate $\mathbf{x}_{i}$ at time $t_{k}$.

$$
p\left(\mathbf{X}_{i}\left(t_{k}\right)=\mathbf{x}_{i}\left(t_{k}\right), \sigma\right)=\frac{1}{N} \sum_{j=0}^{N-1} K\left(\mathbf{x}_{i}-\mathbf{x}_{i_{j}}\left(t_{k}\right)\right)
$$

where $K$ is an isotropic gaussian kernel of size $\sigma$. Thus,

$$
\begin{array}{r}
p\left(\mathbf{X}_{i}\left(t_{k}\right)=\mathbf{x}_{i}\left(t_{k}\right), \sigma\right)=\frac{1}{\sigma \sqrt{2 \pi^{N} \times N}} \sum_{j=0}^{N-1} \cdots \\
\ldots \exp ^{-\frac{1}{2 \sigma^{2}}\left(\mathbf{x}_{i}-\mathbf{x}_{i_{j}}\left(t_{k}\right)\right)^{T}\left(\mathbf{x}_{i}-\mathbf{x}_{i_{j}}\left(t_{k}\right)\right)}
\end{array}
$$

The mean model $\overline{\mathcal{D}}$ and the set of probabilities $\left.p\left(\mathbf{X}_{i}\left(t_{k}\right)=\mathbf{x}_{i}\left(t_{k}\right)\right), i=0 . .2085, t_{k}=[0 . .1]\right\}$ constitute the statistical dynamic model (SDM) of the heart.

\subsection{Results}

This section illustrates some SDM characteristics and its use in 3D+time segmentation tracking.

\subsubsection{SDM coherency}

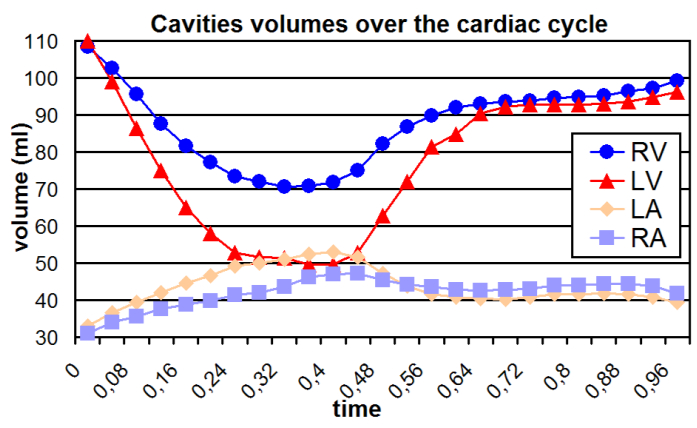

Figure 5. Volumic curves of the 4 chambers of the mean model of the beating heart

Fig. 5 shows the volume of the cavities of the mean dynamic model $\overline{\mathcal{D}}$. The profile of these curves is quite conventional and the computed eject fraction is $60 \%$.

If we consider the variability of one specific landmark at one time point, we can see, in Fig.6, that the bigger the value of the $\sigma$ in the Parzen windowing, the more spread the probability density function. On the other hand, with small values of $\sigma$, the probability function is irregular and localized around the corresponding semi-landmarks in the dataset.

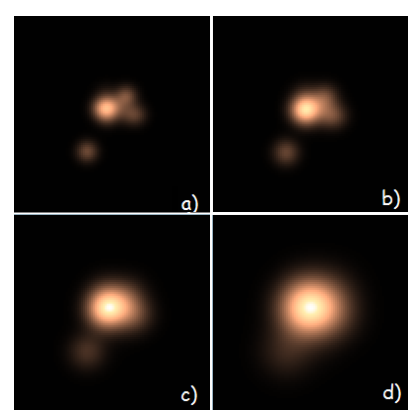

Figure 6. 2D slice of a 3D non linear probability density function for one point at time 0.2 with different variance values : a) $\sigma=2.0$, b) $\sigma=5.0$, c) $\sigma=10.0$, d) $\sigma=20.0$

\subsection{ST-segmentation}

The model can be easily introduced as a regularizing term in cost-function based ST-segmentation and registration algorithms. The a priori knowledge of the shape and 
its evolution are taken into account by minimizing the following energy:

$$
E=E_{\text {data }}+\alpha E_{S D M}
$$

$E_{\text {data }}$ is a data energy term based on a intensity similarity criterion. We try to extract cardiac cavities and their motion for one given time points $t_{k}$. The localization of each semi-landmarks is computed by dynamically sampling their consistent trajectory. This first approach implicitly allows to take into account the temporal evolution of the cardiac shapes. The regularizing energy term is then computed from Eq.(1):

$$
E_{S D M}\left(t_{k}, \sigma\right)=-\frac{1}{P} \sum_{P_{i}} \log \left(p\left(\mathbf{X}_{i}\left(t_{k}\right)=\mathbf{x}_{i}\left(t_{k}\right), \sigma\right)\right)
$$

We introduced this metric term in a free form deformation-based non-rigid multiresolution registration framework. First results tend to show that segmentations are coherent (Fig.7) but parameters' adjustment $(\alpha, \sigma)$ is still currently being experimented.
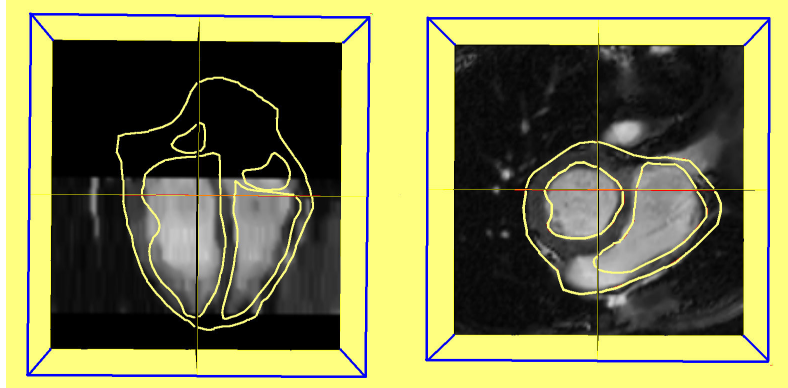

Figure 7. Segmentation result for one subject at time $t=$ 0.1 with $\sigma=10.0$ and $\alpha=5$

\section{Discussion and conclusions}

In this paper, we built a new statistical dynamic model of the heart from the analysis of 16 healthy cases. This model includes ventricles, atria and pericardium. The evaluation of cavities volumes over the full cardiac cycle is realistic and shows the asymmetric temporal behavior of atria and ventricles. Probabilistic framework introduction is well-adapted to model the variability of shape and cardiac chambers behaviors evolution. However with this kind of method, the more cases in the data set, the more relevant the a priori information. Therefore, the enrichment of the data set during as experiments go along is still needed. The $\sigma$ parameter influence is of a particular importance. The bigger the value of the $\sigma$, the more spread the probability density function.
The introduction of the SDM in segmentation and motion tracking algorithms allow to constrain the results according to the dynamic evolution of the cardiac structures. Preliminary results are encouraging but the model registration on the ED image of the test sequence needs to be more accurate to conveniently constraint the algorithm with the SDM. Also, the parameters still have to be adjusted to improve the accuracy of the results.

Temporal windowing taking into account the past and the future in the sequence is to be considered to even increase the temporal consistency.

\section{Acknowledgments}

This work has been supported by the Rhône-Alpes Region through the EURODOC program, and the French Cultural Center at the French Embassy in Helsinki.

\section{References}

[1] Montagnat J, Delingette H. 4D deformable models with temporal constraints: application to 4D cardiac image segmentation. Medical Image Analysis November 2005;9(4):87-100.

[2] Lorenzo-Valdes M, Sanchez-Ortiz G, Mohiaddin R, Rueckert D. Atlas-based segmentation and tracking of 3D cardiac MR Images using non-rigid registration. In MICCAI (2). 2002; 642-650.

[3] Wierzbicki M, Drangova M, Guiraudon G, Petters T. Validation of dynamic heart models obtained using non-linear registration for virtual reality training, planning, and guidance of minimally invasive cardiac surgeries. Medical Image Analysis September 2004;8(3):387-401.

[4] Lötjönen J, Pollari M, Kivistö S, Lauerma K. Correction of movement artifacts from 4-D cardiac short and long-axis MR data. In MICCAI (2). 2004; 405-412.

[5] Grevera G, Udupa J. Shape-based interpolation of multidimensional grey-level images. IEEE Transaction on Medical Imaging December 1996;15(6):881-892.

[6] Lötjönen J, Kivistö S, Koikkalainen J, Smutek D, Lauerma K. Statistical shape model of atria, ventricles and epicardium from short- and long-axis MR images. Medical Image Analysis September 2004;8(3):371-386.

[7] Lötjönen J, Mäkelä T. Elastic matching using a deformation sphere. In MICCAI. 2001; 541.

Address for correspondence:

Bertrand Delhay, CREATIS CNRS UMR 5515, INSERM U630 INSA - Bâtiment Blaise Pascal, 69621 Villeurbanne cedex, France

bertrand.delhay@creatis.insa-lyon.fr 